\title{
INTRODUCCIÓN A LA OPTOELECTRÓNICA (III)
}

\section{INTRODUCTION TO OPTOELECTRONICS (III)}

\section{AUTORES}

Arturo Pérez París: Alumno de la Escuela Politécnica. Universidad de Alcalá arturo.perez@aol.com

\section{CURRÍCULUM VITAE}

Alumno de la Escuela Politécnica de la Universidad de Alcalá de Henares (España). Ingeniero de Soporte Técnico en Kone Elevadores

\section{RESUMEN}

Para esta tercera parte del artículo dejaremos de ser tan físicos para ser más ingenieros, sin menoscabo de una u otra posición. En los procesos industriales se usan muchas veces elementos sensibles a la luz con el fin de controlar diversos sistemas automáticos. Dichos elementos, como ya se dijo, se basan en los principios físicos de fotoemisión y fotoconducción. Por ser los más "normalitos" nos centraremos en los fotosemiconductores.

\section{PALABRAS CLAVE}

Optoelectrónica - Fotoemisión - Fotoconducción 


\begin{abstract}
For this third article we will cease to be so physical for more engineers, without prejudice to either position. In industrial processes are often used light-sensitive elements to control various automated systems. These elements, as noted, are based on physical principles of photoemission and photoconduction. As the most "normalitos" we will focus on fotosemiconductores.
\end{abstract}

\title{
KEY WORDS
}

Optoelectronics - Photoemission - Photoconduction

\section{Introducción}

Para esta tercera parte del artículo dejaremos de ser tan físicos para ser más ingenieros, sin menoscabo de una u otra posición. En los procesos industriales se usan muchas veces elementos sensibles a la luz con el fin de controlar diversos sistemas automáticos. Dichos elementos, como ya se dijo, se basan en los principios físicos de fotoemisión y fotoconducción. Por ser los más "normalitos" nos centraremos en los fotosemiconductores.

El funcionamiento de los diferentes elementos fotosensibles de semiconductores deriva del fenómeno físico por el cual una radiación luminosa cede su energía a los electrones de un sólido, haciendo que estos pasen de la banda de valencia a la de conducción, sin abandonar el material, a diferencia de lo que ocurría con las células fotoeléctricas. Cuando la radiación luminosa incide sobre el material semiconductor, se crean pares electrón - hueco, aumentando el número de portadores de carga 
disponibles y, por tanto, la corriente por el dispositivo, siempre que la energía de la radiación sea suficiente para remontar el ancho de banda prohibida de energía:

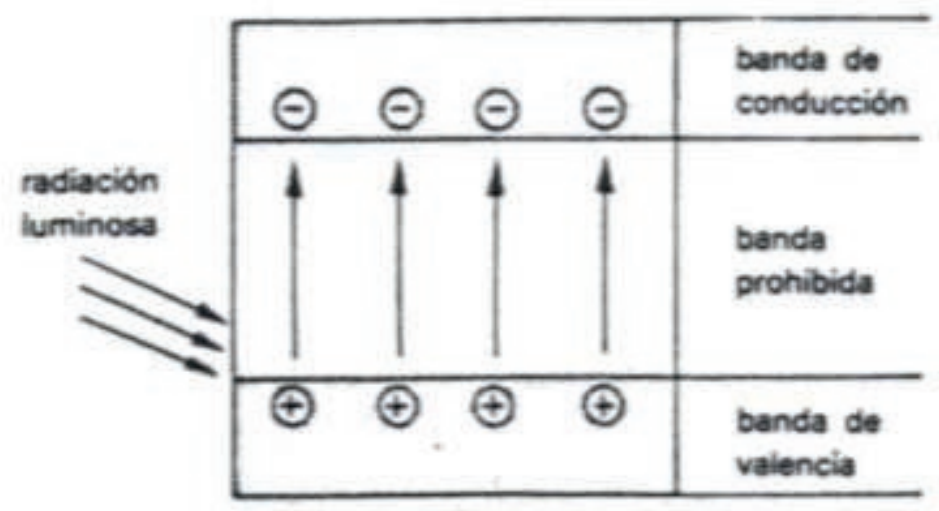

Creación de pares electrón - hueco

La proporción de pares electrón - hueco generados depende de la cantidad de luz absorbida y, por ello, de la geometría del dispositivo y del material empleado, según sea su altura de banda prohibida (cantidad de energía necesaria para pasar de la banda de valencia a la de conducción). Aplicando un campo electríco adecuado capaz de evacuar las cargas generadas, se obtendrá una corriente eléctrica proporcional a la energía luminosa que se absorbe.

\section{Fotosemiconductores de captación}

Un fotodiodo de unión consiste básicamente en una unión NP polarizada en sentido inverso (campo eléctrico en sentido contrario al propio de la unión), de manera que se cree una zona de difusión desprovista de portadores, cuya anchura depende del potencial aplicado: 


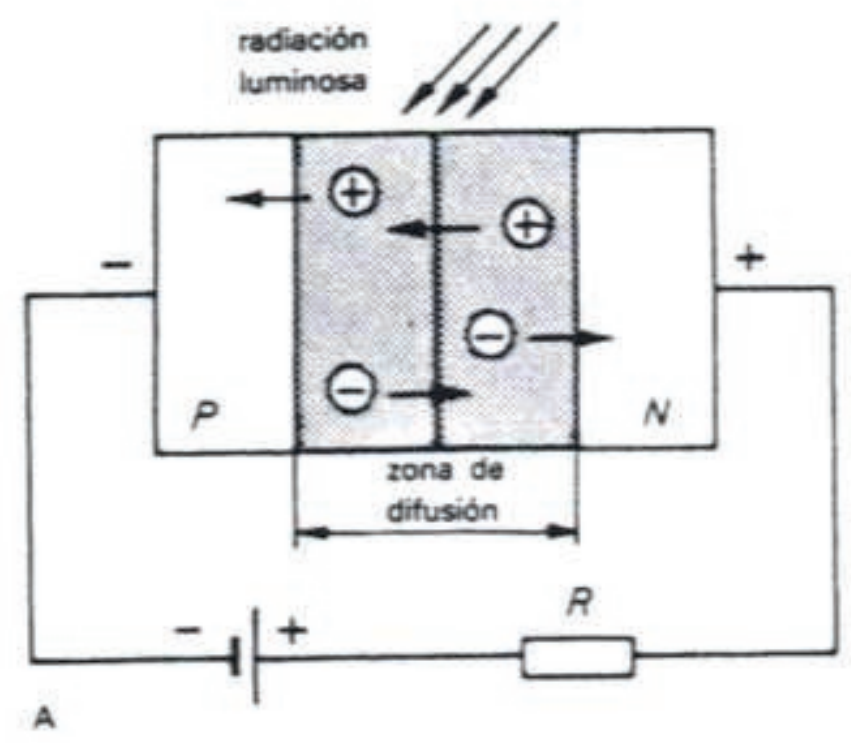

Fotodiodo de unión NP

Cuando no llega ninguna radiación luminosa a esta región, los electrones no tienen energía suficiente para atravesarla y por ello la corriente será prácticamente nula. Cuando la radiación luminosa es de la longitud de onda adecuada e incide en la zona de difusión, se crean pares electrón - hueco que son atraídos por el campo eléctrico aplicado, resultando una corriente inversa por la unión NP proporcional a la energía absorbida y por lo tanto al flujo luminoso que incide sobre la unión, tal y como se muestra en la siguiente curva característica de un fotodiodo genérico: 


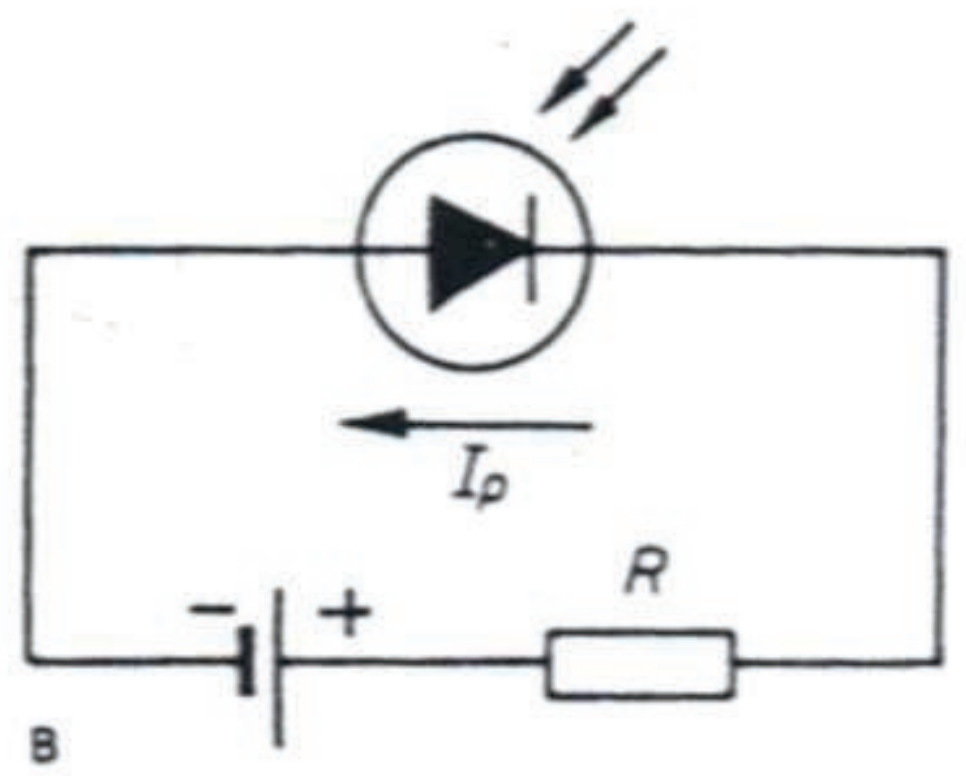

Corriente en un fotodiodo

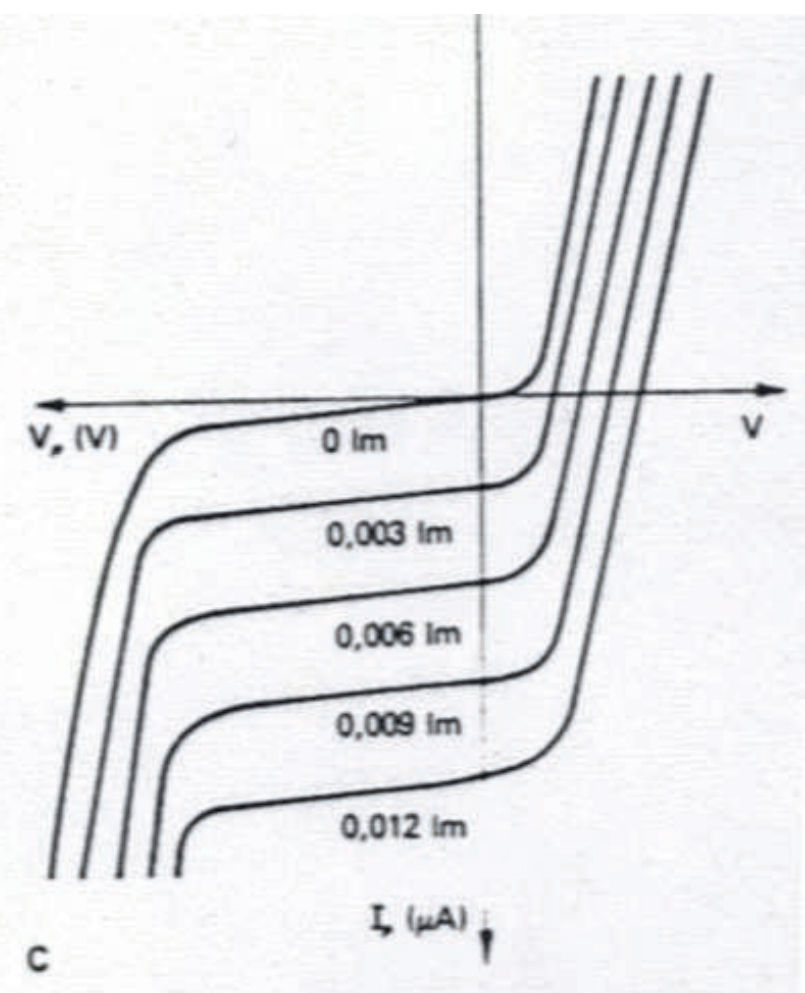

Curvas características de corriente en un fotodiodo en función de su polarización 
Puede verse que el elemento se comporta como un generador de corriente casi constante hasta que se alcanza su tensión de ruptura, tras lo cual se produce una avalancha de portadores y la destrucción del dispositivo. Los fotodiodos pueden fabricarse sobre material base de silicio o de germanio y, en cualquier caso, la unión se forma por el proceso de difusión para obtener una superficie de unión grande y uniforme. La sensibilidad relativa del silicio y la del germanio son similares, quedando la respuesta de este último más próxima a las frecuencias del infrarrojo y teniendo el primero una respuesta más uniforme en la radiación visible. Sin embargo, el silicio presenta una serie de ventajas sobre el germanio tal y como se detallan a continuación:

\begin{tabular}{|l|c|c|}
\hline \multicolumn{1}{|c|}{ Caracteristicas } & Germanio & Silicio \\
\hline $\begin{array}{l}\text { Longitud de onda } \\
\text { de maxima sensibilidad }\end{array}$ & $1500 \AA$ & $900 \AA$ \\
\hline Sensibilidad & 25 a $100 \mathrm{~mA} / \mathrm{m}$ & 30 a $150 \mathrm{~mA} / \mathrm{lm}$ \\
\hline Tiempo de respuesta & 2 a $6 \mu \mathrm{s}$ & 0,2 a $1 \mu \mathrm{s}$ \\
\hline Corriente inversa máxima & 1 a $3 \mathrm{~mA}$ & 3 a $18 \mathrm{~mA}$ \\
\hline Potencia disipable & 30 a $50 \mathrm{~mW}$ & 0,1 a $2 \mathrm{~W}$ \\
\hline Corriente en oscuridad & 0,1 a $1 \mu \mathrm{A}$ & 15 a $50 \mathrm{nA}$ \\
\hline $\begin{array}{l}\text { Deriva térmica } \\
\text { de la corriente }\end{array}$ & $0,7 \%{ }^{\circ} \mathrm{C}$ & $0,1 \%{ }^{\circ} \mathrm{C}$ \\
\hline
\end{tabular}

Tabla de comparación de las características de los fotodiodos

Las corrientes que pueden obtenerse con los fotodiodos son muy limitadas, por ello se recurre a un proceso de amplificación, siendo aquellos meros sensores de muy pequeña potencia. El fototransistor es el dispositivo más simple que puede formarse en la combinación fotodiodo - amplificador. Una manera de ver estos dispositivos, de forma estructural, sería la que a continuación se muestra: 


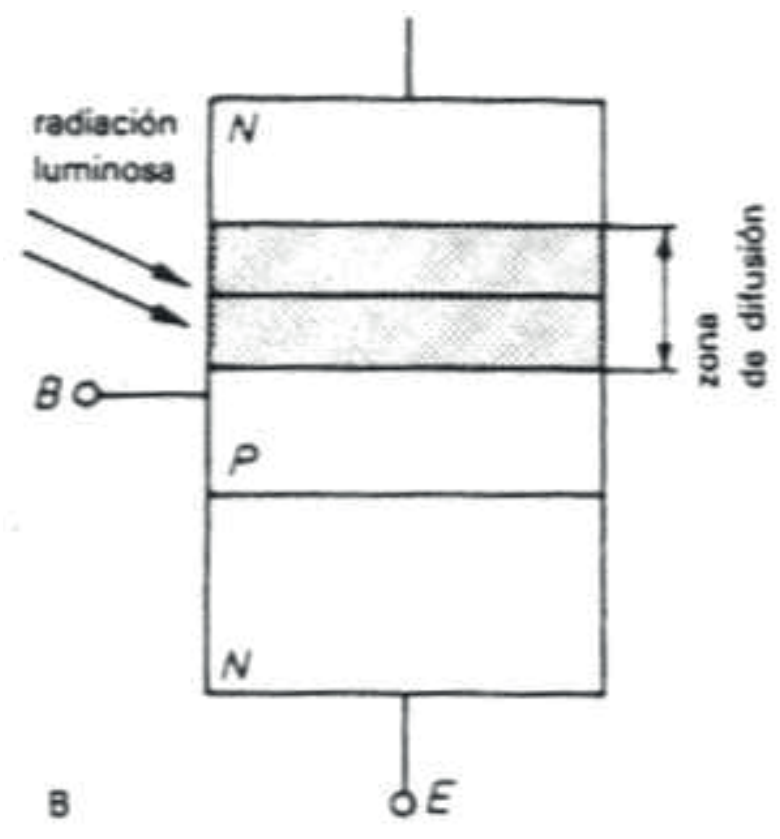

Fototransistor

Como en todo transistor, se polarizará inversamente la unión colector - base, que para estos dispositivos se amplía todo lo posible, con el fin de obtener la máxima superficie de recepción útil de la radiación luminosa. La corriente inversa que, debido a la radiación luminosa, actúa como corriente de base, resulta ser amplificada, según la ganancia de corriente del fototransistor, de modo que la sensibilidad alcanzada por éstos es muy elevada respecto a la de los fotodiodos. La respuesta espectral y las demás características son muy similares a las de los fotodiodos que, como resulta lógico pensar, dependerán del material base utilizado (germanio o silicio).

El circuito equivalente de este dispositivo podría sintetizarse de la siguiente forma: 


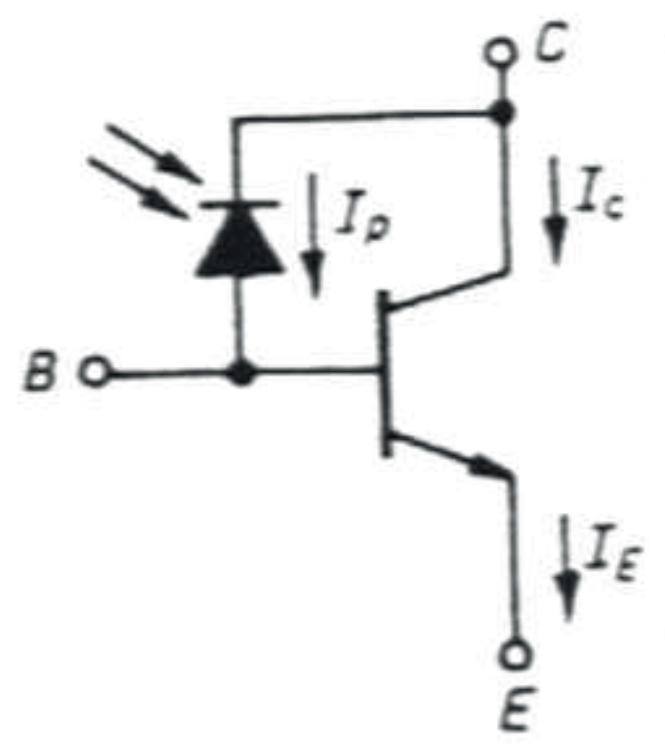

Circuito equivalente de un fototransistor

La corriente de colector depende de la tensión colector - emisor y, por ello, de la radiación luminosa que reciba el dispositivo, tal y como se aprecia a continuación, donde se advierte que el parámetro de corriente de base es sustituido por el flujo luminoso que se recibe:

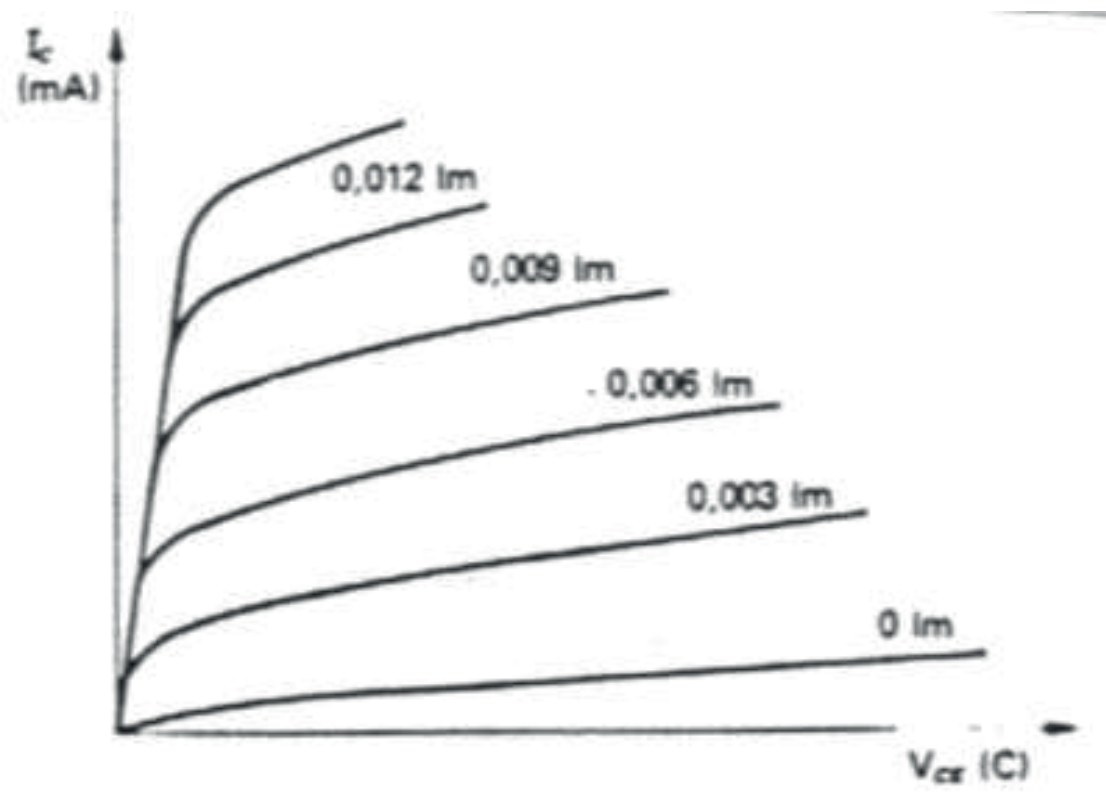


Curvas características de un fototransistor

Para los fototransistores, cuyo terminal de base no es accesible desde el exterior, son correctas las curvas indicadas; mas, cuando se puede acceder a éste, la corriente de colector será la que dan las curvas para una corriente de base igual a Ip + IB siendo Ip la corriente del fotodiodo colector - base e IB la corriente que se introduce desde la fuente exterior.

Cuando los niveles de iluminación son muy bajos o cuando las corrientes de salida necesarias son muy elevadas (sensibilidad elevada), se puede usar un montaje en Darlington o super alfa como el que a continuación se muestra:

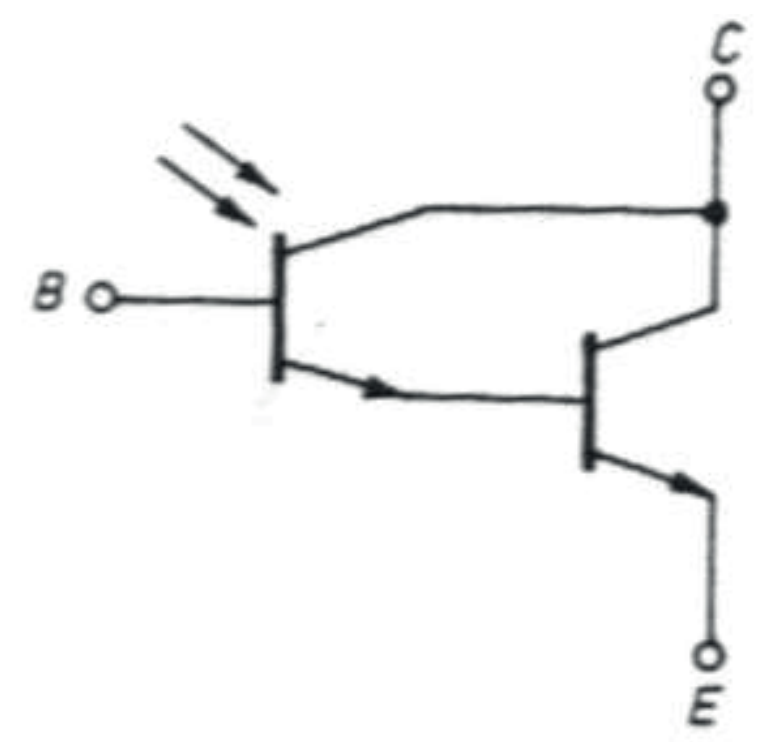

Montaje Darlington, también llamado Super Alfa

Estos montajes se suelen encapsular juntos como si fuese un único componente discreto y su estructura interna podría ser tal como se registra en la figura: 


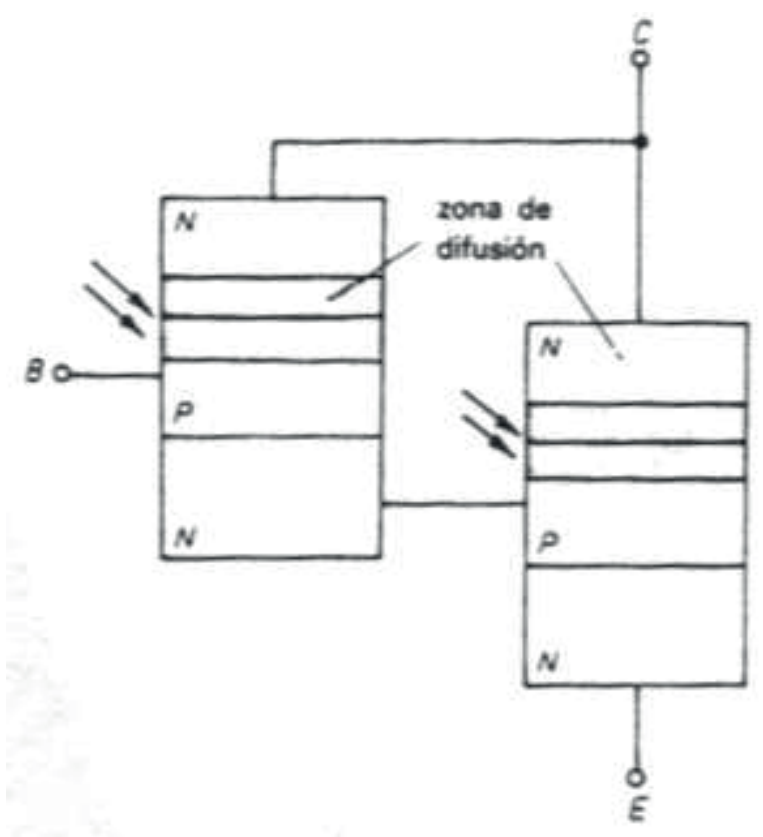

Estructura interna de un montaje Darlington

Se ha visto cómo las respuestas espectrales del silicio y del germanio, utilizados para los fotosemiconductores indicados, correspondían a las radiaciones visibles que entran algo en el rango del infrarrojo, por ello se implementan elementos cuya máxima sensibilidad corresponda a la banda del infrarrojo que, como es obvio, presenta la ventaja de no ser visible al ojo humano, para utilizarlos en todo tipo de automatismos. Como la energía de esta radiación es capaz de crear pares electrón hueco en la zona de difusión del fotosemiconductor, se realizan manufacturas especiales para que la conducción inversa se produzca por avalancha, con lo cual la energía requerida es menor; o se usan materiales de menor altura de la banda prohibida que la correspondiente al silicio o el germanio que responderán únicamente a esta radiación. Estos últimos serán los que más ampliamente se usen para captadores (tanto fotodiodos como fototransistores) de mandos a distancia, etc.

\section{Fotoemisores}


Los diodos emisores de luz son dispositivos semiconductores que basan su funcionamiento en el principio de la electroluminiscencia. También llamados LED, del ingles: Light Emintting Diode, permiten la emisión de luz por la recombinación de los portadores inyectados en la zona de difusión de una unión NP (ya que PN podría sonar obsceno)por medio de una polarización directa, tal y como se muestra a continuación:
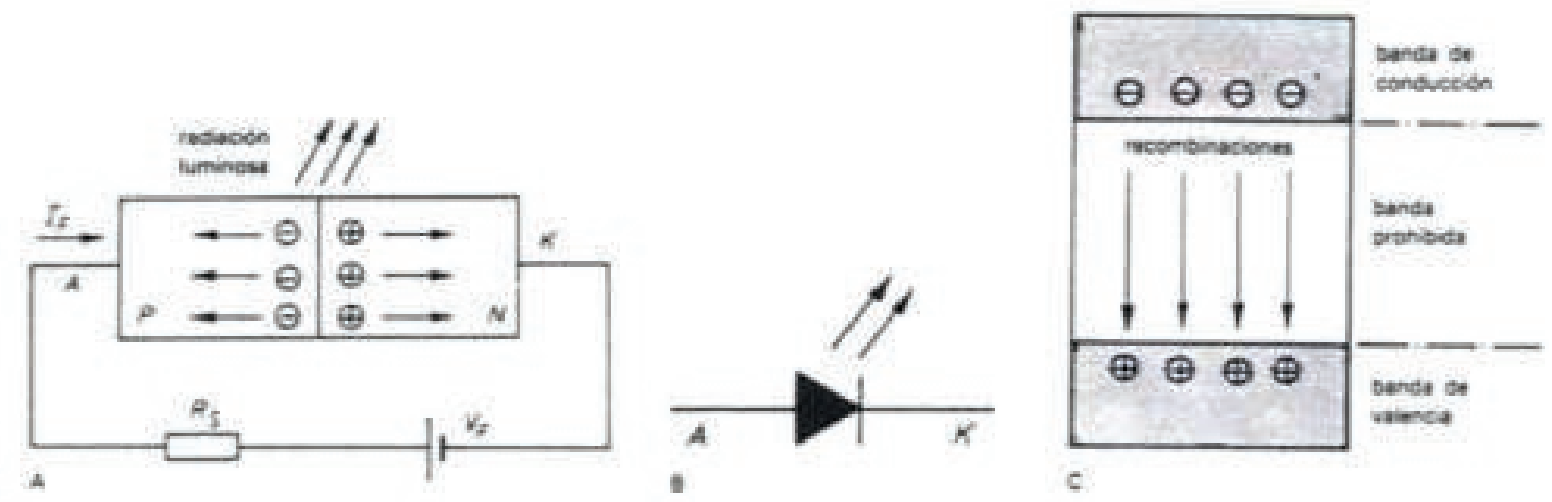

Diodos emisores de luz.

A) Esquema básico. B) Símbolo. C) Recombinación de portadores

Esta recombinación de portadores da lugar a la liberación de una cantidad de energía equivalente a la anchura de la banda prohibida o "gap". Se trata de la recombinación de los electrones inyectados por la fuente de alimentación que ocupan los huecos existentes en la zona de difusión. La energía liberada puede serlo en forma de luz (fotones), de vibraciones de la red atómica del material, con el consiguiente aumento de temperatura, (fonones) o energía cinética comunicada a otros portadores. El tipo de energía liberada depende del material utilizado, de su estructura cristalina, de su pureza y de su forma geométrica. Mediante la combinación adecuada de estos factores, se puede conseguir que la mayor parte de la energía liberada sea en forma de radiación luminosa. El rendimiento entre potencia eléctrica suministrada y la potencia luminosa radiada puede ser del 25\% para los LED. La siguiente gráfica nos 
muestra las longitudes de onda del espectro, indicando el tipo de material semiconductor utilizado para producir emisiones en esa zona. Normalmente en el mercado se encuentran LED's formados por combinaciones de galio, arsénico y fósforo para producir los colores rojo, amarillo, ámbar, verde, azul y emisiones infrarrojas.

El comportamiento de los LED's en un circuito es similar al de cualquier otro diodo de unión, aunque la caída de tensión en directa es mayor debido a que la anchura del gap es también mayor, variando entre 1,2V para el Arseniuro de Galio hasta los $2 \mathrm{~V}$ del Fosfuro de Galio. La tensión en inverso, que son capaces de soportar está entre $5 \mathrm{~V}$ y $25 \mathrm{~V}$, debido a la mayor concentración de impurezas necesaria para una eficiente producción luminosa.

La distribución espacial de la energía luminosa es función del tipo de lente difusora incorporada en la cápsula y del ángulo de desviación con respecto al eje óptico del observador, tal y como se muestra a continuación:
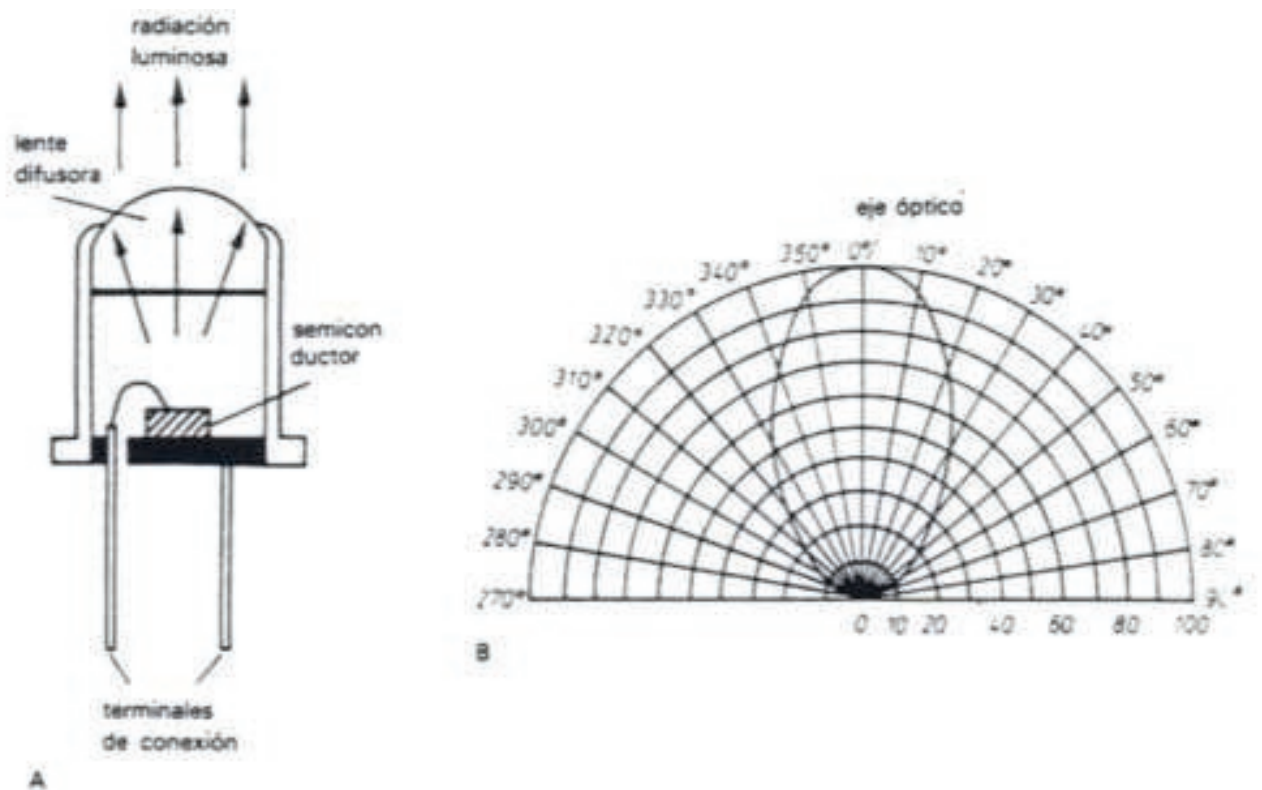

Distribución espacial de la radiación luminosa de un LED. 
A) Estructura física del LED. B) Diagrama de distribución

Los elementos semiconductores descritos pueden usarse de diferentes maneras, para formar sistemas de control o de transmisión de información mediante señales ópticas. Así los elementos emisores y detectores aislados pueden montarse conjuntamente, siempre que su respuesta espectral responda a las mismas longitudes de onda; la regulación y puesta a punto de estos sistemas resultan complicadas, pues es difícil ajustar los términos de la intensidad, la dirección y las distancias de trabajo. Otra forma de uso son los interruptores fotoeléctricos, como el que se muestra en la siguiente figura:

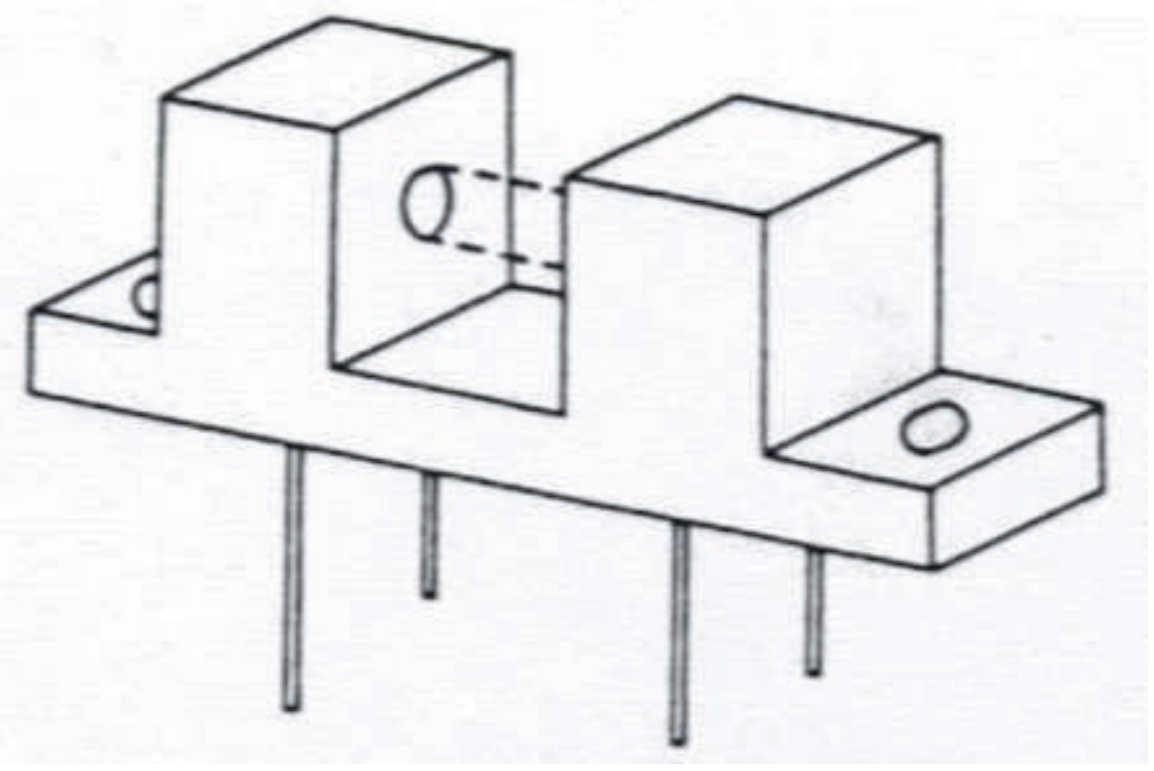

Interruptores fotoeléctricos

Como se ve, se encapsulan un emisor y un detector de características complementarias en módulos perfectamente ajustados. Se suelen usar como finales de carrera, o como generadores de una posición índice de un mecanismo, o para la detección de presencia de un objeto entre su ranura (tarjetas de crédito en un cajero, un disquete en una diquetera, el papel en una impresora, etc.). La mejor forma de utilización conjunta de estos elementos lo constituyen los optoacopladores. Como su 
nombre indica, estos elementos permiten el acoplamiento de una señal eléctrica de entrada en otra señal eléctrica de salida, con un aislamiento galvánico muy elevado entre ambas (del orden de 1015ohmios ). Se construyen en módulos cerrados que no pueden modificarse posteriormente; contienen un diodo emisor, generalmente de infrarrojos, y un elemento detector que puede ser cualquiera de los vistos hasta la fecha. Una muestra genérica de este tipo de elementos vendría dado por:

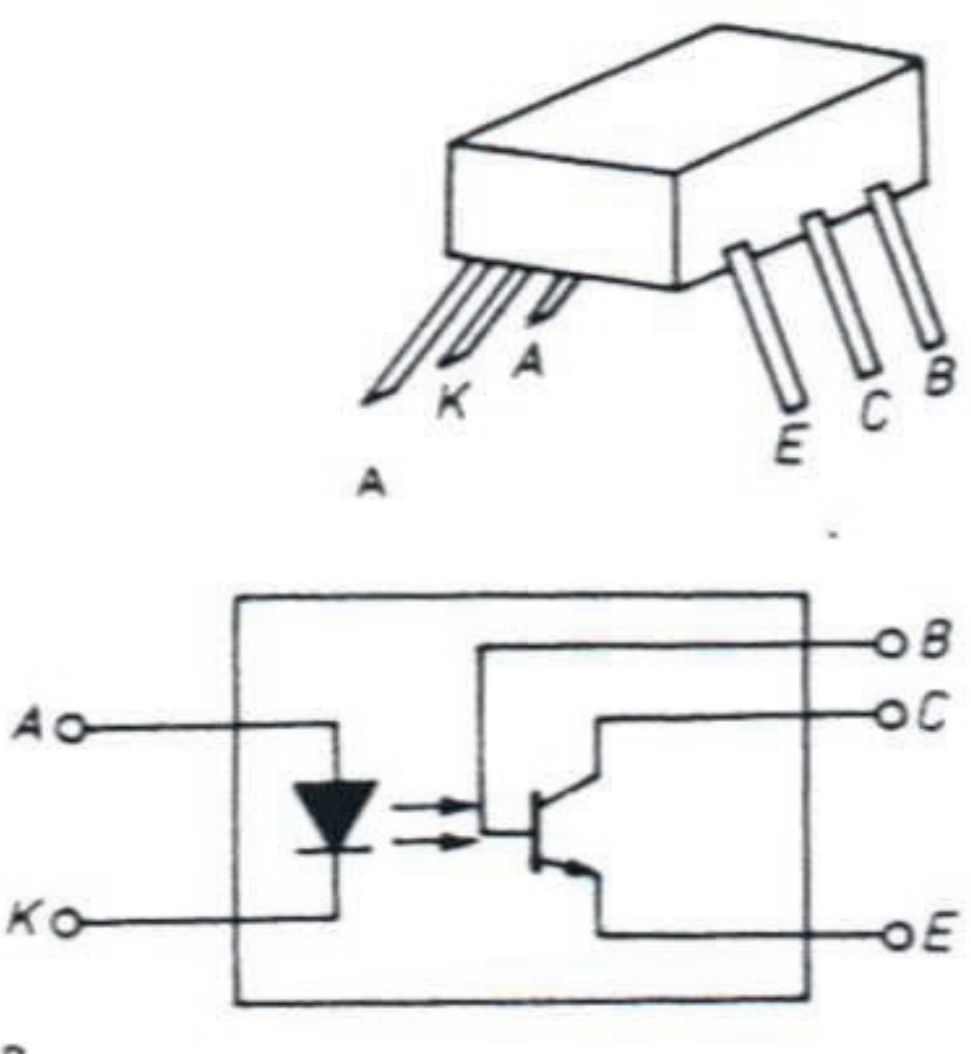

B

Optoacopladores.

A) Aspecto exterior. B) Esquema eléctrico

Aquí termina el presente artículo y todo lo que tenía que decir sobre optoelectrónica y mi pequeño HOMENOTS a D. Cesar Mataix. También quisiera aprovechar la ocasión para agradecer a D. Julio Gutiérrez la supervisión y montaje del presente artículo y a $D^{a}$. Ana María Pérez París (mi hermana), sin cuya ayuda este no hubiese sido posible. Por desgracia (o por fortuna, según se mire) mis exiguos conocimientos 
no llegan más allá, aunque sí que diré que algo me reservo para mí ya que este artículo pretende ser solo una mera introducción (siendo muy generoso) a la asignatura de "Optoelectrónica y fotónica" de mi carrera (I.T.T.S.E.). Si está el lector interesado en este tema, le animo a cursar esta asignatura. En ella realmente adquirirá éstos y muchísimos más conocimientos sobre el tema. Francamente lo recomiendo.

Espero que haya resultado del gusto del lector el presente articulo; si no fue así, desde aquí hago propósito de enmienda para que el próximo artículo salga mejor (eso si no me echan antes por "rollista" ). Si por el contrario le gustó, y despidiéndome una vez más al más puro estilo Shakesperiano, (llegará a convertirse en una tradición esta forma de despedirse) quedemos como amigos y volvamos a encontrarnos donde a la diosa fortuna más le complazca. 